\title{
El cirujano y la subdeterminación en el cáncer de mama. La mamografía, del dilema epistémico al dilema bioético
}

\author{
The surgeon and underdetermination in breast cancer. The mammogram, \\ from epistemic to moral dilemma
}

Alberto Campos*

\footnotetext{
Palabras clave:

Cáncer de mama, mamografía, subdeterminación, dilema moral, bioética.

Key words: Breast cancer, mammogram, underdetermination, moral dilemma, bioethics.
}

\footnotetext{
* Facultad de Medicina, Universidad Nacional Autónoma de México.

\section{RESUMEN}

\section{ABSTRACT}

Frente a imágenes mamográficas sugestivas de malignidad los cirujanos enfrentan un dilema doble, epistémico y moral. Cuando no encuentran coherencia en las guías de práctica y normas oficiales, toman decisiones en solitario y resuelven ambos dilemas pragmáticamente. Las diferentes organizaciones difieren en los criterios de sus recomendaciones para efectuar mamografías de tamizaje. Dado que la subdeterminación entre teoría y evidencia hace que las tesis de las recomendaciones no puedan ser verificadas aisladamente, estas recomendaciones también se hacen con criterios pragmáticos pero no convincentes. De tal manera, los cirujanos tienen que resolver primero el dilema epistémico y luego el dilema moral, y actúan siguiendo sus intuiciones sin considerar las recomendaciones. Utilizan para ello un razonamiento derrotable, racionalmente convincente pero epistemológicamente inválido. El efecto es que sus acciones pueden ser iatrógenas e ir de lo epistémicamente problemático a lo moralmente problemático.
When confronted to mammographic images suggestive of malignancy, surgeons face a double dilemma, epistemic and moral. When they do not find coherence in the clinical guides and official norms, they make decisions on their own and solve both dilemmas pragmatically. Different organizations differ in the criteria of the recommendations and guidelines for breast cancer screening mammograms. Given that the underdetermination between theory and evidence makes it impossible for the thesis of these recommendations to be verified in isolation, they are also made using pragmatic but unconvincing criteria. In such a way, surgeons must first solve an epistemic dilemma, then a moral dilemma, and act on their intuitions. They use a defeasible reasoning, rationally convincing but epistemologically invalid. The effect is that their actions can be iatrogenic and go from the epistemically problematic to the morally problematic.

\section{INTRODUCCIÓN}

U na consecuencia de la tecnología es que mejores mamografías detectan tumores cada vez más pequeños, muchos de los cuales habrían pasado inadvertidos. Ni todos llegarían a una etapa clínica ni serían causa de deceso; algunos habrían sido hallazgos post mortem. Para el cirujano una imagen mamográfica con alteraciones sugestivas de malignidad es un tumor real, su condicionamiento quirúrgico lo induce a actuar con celeridad. Sus pacientes están condicionadas de igual forma. Sin embargo, el problema no es tecnológico, es epistemológico. Aparte de las dificultades propias de la tecno- logía, que crea problemas diferentes de los que se intentaba solucionar, es la metodología del cirujano lo que está en falta; aquello que hace para vincular lo que ve con sus esquemas conceptuales no siempre es coherente.

Los estudios que correlacionan la sobrevida de las pacientes con cáncer (Ca) de mama respecto del diagnóstico por imagen son contradictorios. No toman en cuenta confusores no visibles que se asocian con otras variables. Las diferentes recomendaciones, normas y guías parecen hacerse más con base en ensayo y error que en evidencia epidemiológica.

El cirujano se enfrenta a un dilema doble, epistémico y moral. Frente a imágenes suge- 
rentes de mal pronóstico, ante metodologías que no puede descifrar, como los metaanálisis, y literatura contradictoria y ambigua como las guías de práctica y las normas oficiales, toma sus decisiones en solitario. Resuelve ambos dilemas de manera pragmática. Como consecuencia, sus acciones que pueden ser iatrógenas.

Los objetivos de este artículo son (1) presentar el problema de la multiplicidad de recomendaciones sobre la periodicidad de la mamografía de tamizaje por diferentes agencias y mostrar cómo las nuevas tecnologías, al forzar las conductas terapéuticas, no siempre producen mayor beneficio y sí un mayor daño. Explicar (2), que no hay correlación causal entre imagen y sobrevida, que existe un problema de subdeterminación entre la evidencia radiográfica y la evidencia terapéutica, y que el ajuste en las normas oficiales parece hacerse más como ensayo y error que con base en evidencia. Examinar (3) cómo el cirujano establece clases de cosas y puentes conceptuales entre las imágenes que percibe, las recomendaciones diagnósticas y su núcleo de creencias profundas para tomar decisiones pragmáticas para la acción. Analizar (4) las razones epistémicas y morales del cirujano para tomar decisiones que no son siempre las mejores para las pacientes.

\section{La multiplicidad de recomendaciones}

En los EE. UU. cada vez más cirujanos recomiendan mamografías sin seguir las guías basadas en evidencia (evidence-based guidelines) de la U. S. Preventive Services Task Force (USPSTF), "un grupo independiente, voluntario, de expertos nacionales en la prevención y en la Medicina Basada en Evidencia". ${ }^{1}$ En 2002, la USPSTF recomendaba la mamografía de escrutinio cada año o dos para mujeres de 40 años o más con el argumento de que la detección temprana reduce la mortalidad global entre 16 y $23 \% .^{2}$ El equipo del Nordic Cochrane Center objetó que tanto la mastectomía radical (20\%), como la mastectomía simple y la tumorectomía (30\%) han sido más frecuentes en los grupos tamizados sin evidencia confiable de que la detección temprana reduzca la mortalidad. ${ }^{3}$ De acuerdo con el Nordic Cochrane el efecto de los programas de detección es pequeño, y la proporción entre los efectos benéficos y per- judiciales "muy delicada". ${ }^{4}$ Otros estudios han mostrado que tampoco el examen clínico ni el autoexamen regular de la mama tienen efecto alguno sobre la mortalidad, incluso a pesar de visitas regulares al oncólogo. ${ }^{5-7}$

Las diferentes organizaciones en los EE. UU. difieren en sus recomendaciones para efectuar mamografías. La Asociación Americana de Cancerología (American Cancer Society, ACS) recomienda una mamografía anual a partir de los 45 años (ya no de los 40) y hasta los 55, para continuar luego cada dos años. ${ }^{8}$ La USPSTF también revisó sus guías y relajó la recomendación a un "tamizaje personalizado" para mujeres entre los 40 y 49 años, y luego cada dos años entre los 50 y los $74 . .^{9}$ El argumento es que el exceso de mamografías implica un mayor riesgo de iatrogenia por exceso de diagnóstico. ${ }^{10}$ No obstante, el Congreso Americano de Obstetras y Ginecólogos (American Congress of Obstetricians and Gynecologists, ACOG) todavía recomienda mamografías a mujeres a partir de los 40 años. ${ }^{11}$

En México, el criterio antiguo de la Norma Oficial Mexicana (NOM) Para la Prevención, Diagnóstico, Tratamiento, Control y Vigilancia Epidemiológica del Cáncer de Mama, en 2003, era "anualmente o cada dos años, a las mujeres de 40 a 49 años con dos o más factores de riesgo y en forma anual a toda mujer de 50 años o más." En ella se define el término "factores de riesgo" como "conjunto de condiciones particulares que incrementan la probabilidad de desarrollar una patología" y uno de ellos es "mujer mayor de 40 años." La norma no especifica cómo elegir entre "anualmente o cada dos años"; el criterio queda ambiguo, "por indicación médica."12

La última NOM, del 2011 es más moderada; la mamografía "se recomienda en mujeres aparentemente sanas de 40 a 69 años de edad, cada dos años [y] en mujeres menores de 40 años, no se recomienda la mastografía de tamizaje." ${ }^{13}$ La recomendación es ambigua a partir de los 70 años, "como parte del seguimiento de mujeres con antecedente personal de cáncer de mama y (de nuevo) por indicación médica."

A pesar del cambio hacia la moderación tanto de la ACS como de la USPSTF, otro estudio reveló que en los EE. UU. una gran proporción de médicos (81\%) recomienda 
mamografías de tamizaje en mujeres entre 40 y 44 años, aun cuando el daño es más probable que el beneficio, porque "los clínicos deciden con base en las guías de aquella asociación en la que más confían." ${ }^{14}$ Es interesante que no muchos médicos sigan las recomendaciones de la USPSTF, pretendidamente más "evidencebased transparent y libres de conflicto". De acuerdo con una encuesta nacional, un $88 \%$ de los médicos "inexplicablemente confía en otras guías" y recomienda mamografías de rutina para mujeres entre 40 y 45 años, y un $67 \%$ recomienda mamografías para mujeres de 75 años y más, a pesar de que la mitad de las mujeres con más de 10 mamografías, termina por tener un resultado falso positivo que resulta en más mamografías, resonancias magnéticas y biopsias innecesarias. ${ }^{15}$

El potencial de reducción de la mortalidad por Ca de mama parecería estar asociado con una menor incidencia en la detección de tumores grandes. Sin embargo, un estudio que correlacionó tamaño tumoral, sobrediagnóstico y efectividad de la mamografía, con datos del Programa de Vigilancia, Epidemiología y Resultados Finales (Surveillance, Epidemiology, and End Results, SEER) ${ }^{16}$ concluye que ha habido un mayor exceso de diagnósticos falsos positivos que de tumores destinados a crecer de haber permanecido ocultos, y que la magnitud del sobrediagnóstico es mayor de lo que generalmente se reconoce. ${ }^{17} \mathrm{El}$ mismo estudio demuestra que la disminución de la mortalidad específica de acuerdo con el tamaño se debe a una mejoría en la terapia sistémica, no al aumento en la detección de tumores pequeños.

\section{La subdeterminación}

La noción de subdeterminación en la filosofía de la ciencia establece que la evidencia por sí misma no permite elegir una teoría que explique totalmente esa evidencia. La correlación entre una mamografía y un proceso tisular no permite establecer una relación causal. La imagen por sí misma no refleja el comportamiento biológico del tumor ni su grado de diferenciación celular, ni determina la sobrevida de una paciente.

Podemos saber que causas y efectos ocurren más o menos juntos, regular y constante- mente, pero de esto no se sigue que esa simple conjunción de eventos suponga la causalidad de uno por el otro. Pensar así sugiere la llamada Visión de la Regularidad de la Causalidad, en la que contigüidad espacio temporal, sucesión y regularidad son elementos constitutivos de la causalidad. ${ }^{18}$ Esa sucesión y continuidad de dos eventos, la costumbre de verlos en conjunción constante, crea una impresión de causalidad, pero no permite explicar cómo es esa relación causal. ${ }^{19}$

La predicción del fenómeno objeto de la investigación no deriva de una proposición sola. De acuerdo con Pierre Duhem, los experimentos producen observaciones de fenómenos acompañadas de interpretaciones diversas, pero no es posible someter a prueba experimental una hipótesis aislada, sino sólo un grupo de hipótesis auxiliares que interactúen en conjunto para explicar el fenómeno. Si un experimento no concuerda con las predicciones, al menos una de sus hipótesis es inaceptable y debe ser cambiada, pero el experimento no designa cuál. Si el fenómeno (el resultado) esperado no se produce, lo único que el estudio dice es que entre las proposiciones usadas para predecirlo hay al menos un error, pero no dice dónde está. ${ }^{20}$

La subdeterminación indica que siempre habrá un desajuste entre teoría y evidencia, que las hipótesis causales no pueden ser verificadas aisladamente sino en contexto con un cuerpo de creencias. ${ }^{21}$ De tal manera, una teoría está subdeterminada incluso respecto de todas las observaciones posibles, en virtud de que existen eventos observables pero aún no observados, de que los criterios observacionales de las proposiciones teóricas comúnmente son "flexibles y fragmentarios" y de que pueden ser empíricamente equivalentes pero lógicamente incompatibles. ${ }^{22}$

Ningún estudio nos dice exactamente qué proposición debemos revisar o rechazar como equivocada porque los criterios teóricos que se prueban representan un rango en la solidez de la evidencia. Así las cosas, la proposición "existe una correlación entre el tamaño del tumor en el momento del diagnóstico y la sobrevida en el cáncer de mama" no puede ser verificada. Tomada independientemente de otras hipótesis, ese enunciado es insuficiente para determinar 
cualquier teoría que relacione detección temprana con pronóstico. Hay hipótesis biológicas acerca de mutaciones genéticas, diferenciación celular, invasividad local y capacidad metastásica, e hipótesis terapéuticas como la extensión (in)suficiente de la cirugía, y el complemento con quimio y radioterapia.

La mamografía es solamente una búsqueda de pequeñas anormalidades anatómicas, y en los diversos metaanálisis de sobrevida de Ca de mama hay otras hipótesis empíricamente equivalentes pero lógicamente incompatibles con el tamaño del tumor en el momento del diagnóstico. De tal manera, ante una imagen mamográfica sugestiva de alteración anatómica, nuestra respuesta también está subdeterminada por la imagen. Requerimos de otras hipótesis para dar congruencia al diagnóstico que necesitamos formar y poder diseñar un proyecto terapéutico para esa paciente. La mamografía no dice cómo estructurar ese plan.

\section{Clases de cosas, puentes conceptuales y decisiones pragmáticas}

Demostrar cómo constituimos clases de cosas es uno de los problemas más difíciles de la epistemología. Cómo, a partir de las reconstrucciones psicológicas de nuestras percepciones, establecemos lo que entendemos como conocimiento. Cómo llega el cirujano a construir objetos mentales, por ejemplo, los tumores, a partir de las reconstrucciones digitales de las radiaciones que pasan a través de tejidos mamarios de densidades diferentes. Y luego cómo, a partir de esas reconstrucciones, forma conceptos tales como sobrevida, pronóstico y aún otros como curación o iatrogenia.

La constatación sensorial de aquello que percibimos es la demostración empírica más sencilla, pero la mayor parte de nuestro conocimiento es indirecta; de los fenómenos que no se ven, sólo podemos ver sus efectos. Formamos puentes de ideas entre nuestras percepciones sensoriales y las construcciones más o menos racionales (o intuitivas) de nuestros conceptos teóricos.

El cambio de las guías para recomendar una menor frecuencia de tamizaje resulta contrario a conceptos previos de cirujanos y pacientes. En ese contexto es muy difícil separar problemas descriptivos (el tamaño del tumor) de problemas normativos (la periodicidad de la mamografía de acuerdo con la edad de la paciente). Una cosa es que los datos producidos por un metaanálisis tengan cierto grado de adecuación empírica y otra cosa es que sean verdaderos. Por eso las consideraciones que nos guían para ajustar nuestro cuerpo existente de conocimientos con la percepción sensorial de las imágenes son de alguna forma pragmáticas, ni puramente lógicas ni puramente psicológicas.

\section{Razones epistémicas y morales}

Frente a esos tumores pequeños sobrediagnosticados por tecnologías cada vez más finas, el cirujano tiene una intuición de riesgo. Tiene la impresión, genuina, de que es mejor actuar que no actuar, a pesar de que no exista evidencia concluyente, a pesar de que la evidencia de curación esté muy subdeterminada por el tamaño del tumor en el momento del diagnóstico. Sin importar, en su intuición, ninguna de las demás variables, la espera no es opción.

El cirujano experimenta entonces un riesgo epistémico. Subjetivamente sabe que no sabe; objetivamente no conoce la probabilidad de que ese tumor progrese local y metastásicamente hasta la muerte de la paciente. Puesto que las probabilidades relevantes para tomar una decisión le son desconocidas, confunde su estado de incertidumbre con riesgo, confunde su creencia con verdad. El problema es saber dónde está la verdad, creer en la adecuación empírica de sus intuiciones como si fueran verdad. El problema es que la verdad no puede conocerse, que las probabilidades no son certezas; entonces, para resolver su dilema epistémico, toma una decisión pragmática en la ignorancia. Eso tiene el efecto de que la intuición se invierte, el dictum "lo primero es no dañar" es sobreseído por la acción. Ahora "lo primero es actuar", quitar el tumor, lo demás viene después. Este escenario se construye sobre la base de mecanismos mentales y premisas lógicamente mal reglamentadas o dejadas a esas intuiciones supuestamente correctas pero empíricamente inadecuadas.

Para resolver el dilema moral, los cirujanos proveen y mezclan las justificaciones habituales de los estudios innecesarios; algunas son 
epistemológicas, otras altruistas, las últimas puramente egoístas. Entre las justificaciones epistemológicas, vimos arriba, la idea de que ante la incertidumbre es mejor hacer algo que no hacer nada, mejor quitar un tumor que no quitarlo. Entre las altruistas, la convicción de que el cuidado de las pacientes significa ir por encima de las recomendaciones, con el argumento insuficientemente sustentado de que ellas mismas prefieren mayor cantidad de pruebas para mayor tranquilidad, en vez de dejar pasar las cosas. Las justificaciones puramente egoístas se fundamentan en el miedo al litigio por negligencia y en el hecho de que, a fin de cuentas, la paciente se hará operar por otro cirujano, quien obtendrá los beneficios económicos, más aún cuando sea el caso de que no ella sino alguna aseguradora pague los gastos. En este caso la justificación tiene el atenuante añadido de que no desembolsa la paciente sino una empresa que, además, le condiciona sus honorarios.

Éstas pueden ser algunas explicaciones, aunque no exhaustivas, del impulso de biopsiar imágenes que en la memoria del cirujano corresponden a tumores en el sentido más palpable. El cirujano está seguro de contrastar esas imágenes con el tumor que tocará durante la intervención y que luego mostrará a la paciente. Lo que no puede explicar, ni lógica ni empíricamente, es la noción que tiene de hacer un bien a la paciente por el sólo hecho de extirpar un tumor de inmediato, independientemente de la estirpe, el grado de diferenciación tisular, la respuesta que ésta tendrá al tratamiento complementario, etc. Pero, además, las justificaciones tienen ventajas pragmáticas; sustituyen las discusiones informadas sobre los riesgos y beneficios de la mamografía y evitan la posibilidad de que la paciente decida posponer la decisión o acudir con otro. Esas ventajas no justifican la iatrogenia.

Las instituciones y asociaciones tampoco pueden explicar, lógica, empírica o convincentemente, sus criterios para modificar las recomendaciones sobre la periodicidad de las mamografías. Toman en cuenta el incremento de diagnósticos falsos positivos, de iatrogenias por exceso de biopsias confirmatorias y de cirugías por tumores pequeños que las recomendaciones previas fomentaron, $y$ el efecto del aumento de esos procedimientos en los costos de la atención de la salud, pero sin una correlación causal demostrable entre frecuencia del estudio y sobrevida.

El criterio de evitar procedimientos innecesarios es válido, importante, pero no el único. El objeto de este análisis es precisamente su poco éxito, pues los procedimientos han aumentado a pesar de las recomendaciones. No han convencido contra la lógica previa de la bondad de una mayor frecuencia de exámenes, una variante de la ley (y en este caso una falacia heurística) de los grandes números. La ley afirma que la precisión de una serie estadística tiende a mejorar con un mayor número de ensayos, y que extrapolada a la mayor frecuencia de mamografías con mejores aparatos produciría una mayor detección de tumores más pequeños. Pero de esto no se sigue que por ese solo hecho, sin más, la sobrevida de las pacientes con Ca de mama sea más larga. Las recomendaciones previas fueron hechas con base en esta falacia sin tomar en cuenta los factores confusores mencionados en la introducción. En la percepción del cirujano una ley estadística se convirtió, por recomendación, en una falacia lógica que éste tiene por verdadera; por eso no puede anular su creencia previa. En su núcleo de creencias profundas un tumor benigno es el tumor en una bandeja.

El argumento de que la sobrevida se alarga entre más temprana la detección del tumor de mama es entonces un razonamiento derrotable, la veracidad de sus premisas es racionalmente convincente pero el argumento es epistemológicamente inválido y su conclusión falsa.

\section{CONCLUSIÓN}

La subdeterminación de la teoría por la evidencia disponible y las conexiones causales anómalas entre hipótesis incompatibles, como la relación del tamaño de un tumor con su pronóstico de vida, puede coaccionar en ocasiones a una toma de decisiones pragmáticas. A pesar de haberse atenuado las recomendaciones previas sobre la frecuencia de las mamografías de tamizaje, se observa paradójicamente un aumento en la cantidad de mamografías, biopsias y mastectomías por diagnósticos falsos positivos. Ante una lesión 
que se sospecha maligna, la conducta expectante resulta contraintuitiva.

Frente a imágenes sugerentes de mal pronóstico, metodologías que no puede descifrar, como los metaanálisis, y literatura contradictoria y ambigua como las guías de práctica y las normas oficiales, frente a sus dilemas epistémico y moral, resuelve ambos en solitario y de manera pragmática. Como consecuencia, sus acciones pueden ser iatrógenas.

Esas intenciones pueden ir de lo más altruista a lo más egoísta. Sus acciones pueden distribuirse en un continuo, de lo bien intencionado pero epistémica y éticamente mal justificado a lo francamente inmoral.

\section{REFERENCIAS}

1. USPSTF (U.S. Preventive Services Task Force) 2014. Grade Definitions After July 2012. What the Grades Mean and Suggestions for Practice. En: Grade Definitions. U.S. Preventive Services Task Force. October 2014. En: http://www. uspreventiveservicestaskforce.org/Page/Name/gradedefinitions. Consultado el 21/04/2016.

2. Humphrey LL, Helfand M, Chan BKS, et al. Breast cancer screening: a summary of the evidence for the U.S. Preventive Services Task Force. Ann Intern Med. 2002; 137: 347-360.

3. Olsen O, Gøtzsche PC. Cochrane review on screening for breast cancer with mammography. Lancet. 2001; 358: 1340-1342.

4. Gøtzsche PC, Olsen O. Is screening for breast cancer with mammography justifiable? Lancet. 2000; 355: 129-134.

5. Kerlikowske K. Efficacy of screening mammography: a meta-analysis. JAMA. 1995; 273: 149-154.

6. Semiglazov VF, Moiseenko VM, Protsenko SA, et al. Preliminary results of the Russia (St Petersburg)/WHO Program for the Evaluation of the Effectiveness of Breast Self-Examination. Vopr Onkol. 1996; 42: 49-55. En: Gøtzsche \& Olsen, 2000.

7. Thomas DB, Gao DL, Self SG, et al. Randomized trial of breast self-examination in Shanghai: methodology and preliminary results. J Natl Cancer Inst. 1997; 89: 355-365. En: Olsen y Gøtzsche, 2001.

8. Oeffinger KC, Fontham ETH, Etzioni R, et al; American Cancer Society. Breast cancer screening for women at average risk: 2015 guideline update from the American Cancer Society. JAMA. 2015; 314: 1599-1614. doi: 10.1001/jama.2015.12783.

9. Siu AL, U.S. Preventive Services Task Force. Screening for breast cancer: U.S. Preventive Services Task Force Recommendation Statement. Ann Intern Med. 2016; 164: 279-296. doi: 10.7326/M15-2886.
10. Radhakrishnan A, Nowak SA, Parker AM, Visvanathan K, Pollack CE. Physician breast cancer screening recommendations following guideline changes: results of a national survey. JAMA Internal Medicine. (E1, E2) Publicado en línea. Abril 10, 2017.

11. American College of Obstetricians-Gynecologists. Practice bulletin no. 122: Breast cancer screening. Obstet Gynecol. 2011; 118: 372-382. doi: 10.1097/ AOG.0b013e31822c98e5.

12. Secretaría de Salud. Norma Oficial Mexicana NOM041-SSA2-2002, para la prevención, diagnóstico, tratamiento, control y vigilancia epidemiológica del cáncer de mama. Diario Oficial de la Federación (DOF) 17 de septiembre, 2003.

13. Secretaría de Salud. Norma Oficial Mexicana NOM041-SSA2-2011, para la prevención, diagnóstico, tratamiento, control y vigilancia epidemiológica del cáncer de mama. DOF, 9 de junio de 2011.

14. Peterson EB, Ostroff JS, DuHamel KN, et al. Impact of provider-patient communication on cancer screening adherence: a systematic review. Prev Med. 2016; 93: 96-105. doi: 10.1016/j.ypmed.2016.09.034.

15. Grady D, Redberg RF. Physician adherence to breast cancer screening recommendations. JAMA Intern Med. Publicado en línea, abril 10, 2017. doi: 10.1001/ jamainternmed.2017.0458.

16. SEER*Stat Database: (1975-2012). Bethesda, MD: National Cancer Institute Surveillance Research Program, 2015 (http://www .seer .cancer .gov).

17. Welch GH, Prorock PC, O'Malley AJ, Kramer BS. Breast-cancer tumor size, overdiagnosis, and mammography screening effectiveness. N Engl J Med. 2017; 375: 1438-1447.

18. Psillos S. Regularity theories. En: Beebee H, Hitchcock Ch, Menzies P. (eds.). The Oxford Handbook of Causation. Oxford University Press; 2009. p. 155 y ss.

19. Hume D. A treatise of human nature. Ed. L.A. SelbyBigge. Oxford, Clarendon Press 1739 reimpr. 1888. Libro I, Parte III, §, pp. 170 y ss.

20. Duhem P. [1914] The Aim and Structure of Physical Theory, 2a. ed. (trad. P. W. Wiener de la v.o.: La Théorie Physique: Son Objet et sa Structure (Paris: Marcel Riviera \& Cie.), Princeton, NJ, Princeton University Press; 1954. p.187.

21. Quine WV. Two dogmas of empiricism. En: From a logical point of view. (2a. ed.) Harvard University Press; 1961. p. 20-46.

22. Quine $W V$. On the reasons for indeterminacy of translation. The Journal of Philosophy. 1970; 67: 178183.

\section{Correspondencia:}

Alberto Campos

Facultad de Medicina, Universidad Nacional

Autónoma de México.

Ciudad Universitaria, 04510,

Ciudad de México.

E-mail: alberto_campos@hotmail.com https://orcid.org/0000-0001-5811-1908 